Supplement of Solid Earth, 9, 1517-1533, 2018

https://doi.org/10.5194/se-9-1517-2018-supplement

(C) Author(s) 2018. This work is distributed under

the Creative Commons Attribution 4.0 License.

(c) (1)

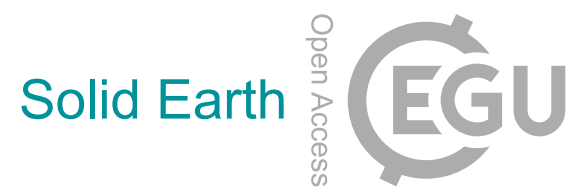

Supplement of

\title{
The effect of rock composition on muon tomography measurements
}

Alessandro Lechmann et al.

Correspondence to: Alessandro Lechmann (alessandro.lechmann@geo.unibe.ch)

The copyright of individual parts of the supplement might differ from the CC BY 4.0 License. 


\section{Energy loss in rocks - mass averaging approach}

To obtain an energy loss equation for rocks, a similar procedure as for forming minerals out of elements can be applied. Starting from Eq. (B5) we consider the energy loss for a rock as mass weighted average of the energy losses of its mineral constituents

$\left\langle\frac{d E_{\text {rock }}}{d \chi}\right\rangle=\sum_{j} q_{j}\left\langle\frac{d E_{\text {mineral,j }}}{d \chi}\right\rangle$

where $q_{j}$ are the mass fractions of the $\mathrm{j}$-th mineral within the rock, analogous to Eq. (B6),

$\mathrm{q}_{\mathrm{j}}=\frac{n_{j} A_{j}}{\sum_{l} n_{l} A_{l}}=\frac{m_{\text {mineral }, j}}{m_{\text {rock }}}$

Analogue to the mineral case we can now define new average energy loss parameters for the rock. The formula for mass density, given the weight fractions, is

$\rho_{\text {rock }}=\frac{1}{\sum_{j} \frac{\mathrm{q}_{\mathrm{j}}}{\rho_{\text {mineral }, j}}}$

The average $\{Z / A\}$ is given by

$\left\{\frac{Z}{A}\right\}=\sum_{j} \mathrm{q}_{\mathrm{j}}\left\langle\frac{Z}{A}\right\rangle_{j}$

and similarly, the average $\left\{Z^{2} / A\right\}$ can be calculated according to

$\left\{\frac{Z^{2}}{A}\right\}=\sum_{j} q_{j}\left\langle\frac{Z^{2}}{A}\right\rangle_{j}$

The rock's mean excitation energy is

$\ln \{I\}=\frac{\sum_{j} q_{j}\left(\frac{Z}{\mathrm{~A}}\right\rangle_{j} \ln \langle I\rangle_{j}}{\sum_{l} \mathrm{q}_{j}\left(\frac{Z}{\mathrm{~A}}\right\rangle_{l}}$

The only difference between the rock calculation and the mineral calculation enters in the calculation of the plasma energy. While in the mineral case we were advised to use Eq. (B11) instead of what would naturally follow from the weighted average in Eq. (B5), we prefer to use the weighted average, Eq. (S1),

$\ln \left\{\hbar \omega_{p}\right\}=\frac{\sum_{j} \mathrm{q}_{j}\left\langle\frac{Z}{\mathrm{~A}}\right\rangle_{j} \ln \left\langle\hbar \omega_{p}\right\rangle_{j}}{\sum_{l} \mathrm{q}_{\mathrm{l}}\left\langle\frac{Z}{\mathrm{~A}}\right\rangle_{l}}$

for the case of rocks. The reason for this lies in the fact that the density effect operates on a nanometric scale, whereas minerals, in general have sizes between several micrometres and a few centimetres. In the case of a mineral compound, the molecular structure is also on a nanometric scale.

These parameters can then be rearranged into an ionisation loss term for a rock

$\left\{a\left(E, \rho_{\text {rock }}, A, Z\right)\right\}=K\left\{\frac{Z}{A}\right\} \frac{1}{\beta^{2}}\left[\frac{1}{2} \ln \left(\frac{2 m_{e} c^{2} \beta^{2} \gamma^{2} Q_{\max }(\mathrm{E})}{\{I(Z)\}^{2}}\right)-\beta^{2}-\frac{\delta\left(\rho_{\text {rock }},\left\{\frac{Z}{A}\right\}\right)}{2}+\frac{1}{8} \frac{Q_{\max }^{2}(\mathrm{E})}{\left(\gamma \mathrm{m}_{\mu} c^{2}\right)^{2}}\right]+\Delta\left|\frac{d E}{d X}\right|\left(\left\{\frac{Z}{A}\right\}\right)$.

Like Eq. (B14) the radiative losses can be rewritten as a weighted average of the mineral radiative losses

$\{b\}=\sum_{j} q_{j}\langle b\rangle_{j}$. 
Equations. (S8) and (S9) can then be joined together to form again a similar term to Eqs. (B1) and (B15),

$-\left\{\frac{d E_{\text {rock }}}{d x}\right\}=\rho_{\text {rock }} *(\{a\}+E *\{b\})$,

the energy loss equation for rocks. 


\section{Calculations for an alternative flux model}

In this supplement, we present the resulting figures of our calculations for a flux model by Tang et al. (2006). Similar to the flux model by Reyna (2006), we attributed to both models a systematic error of $\pm 15 \%$. Essentially, the lithology-specific effect on the flux remains the same for all lithologies. Furthermore, the same conclusions can be drawn, i.e. that it is only safe to use the standard rock approximation up to $300 \mathrm{~m}$ thickness and that limestone and basalt deviate the most from the density modified standard rock flux.

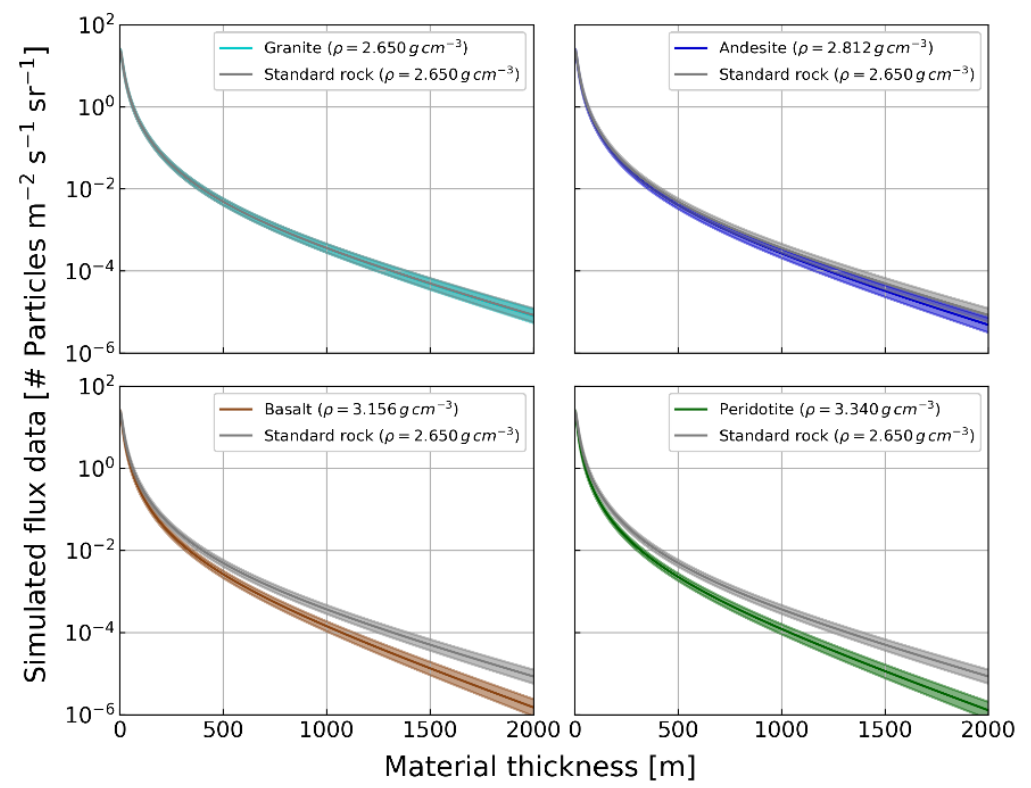

Figure S2-1: Simulated muon intensity vs. thickness of the four igneous rocks from Table 1 and standard rock. The mean flux is indicated by a bold line and $1 \sigma$ bounds are indicated by the shaded area.

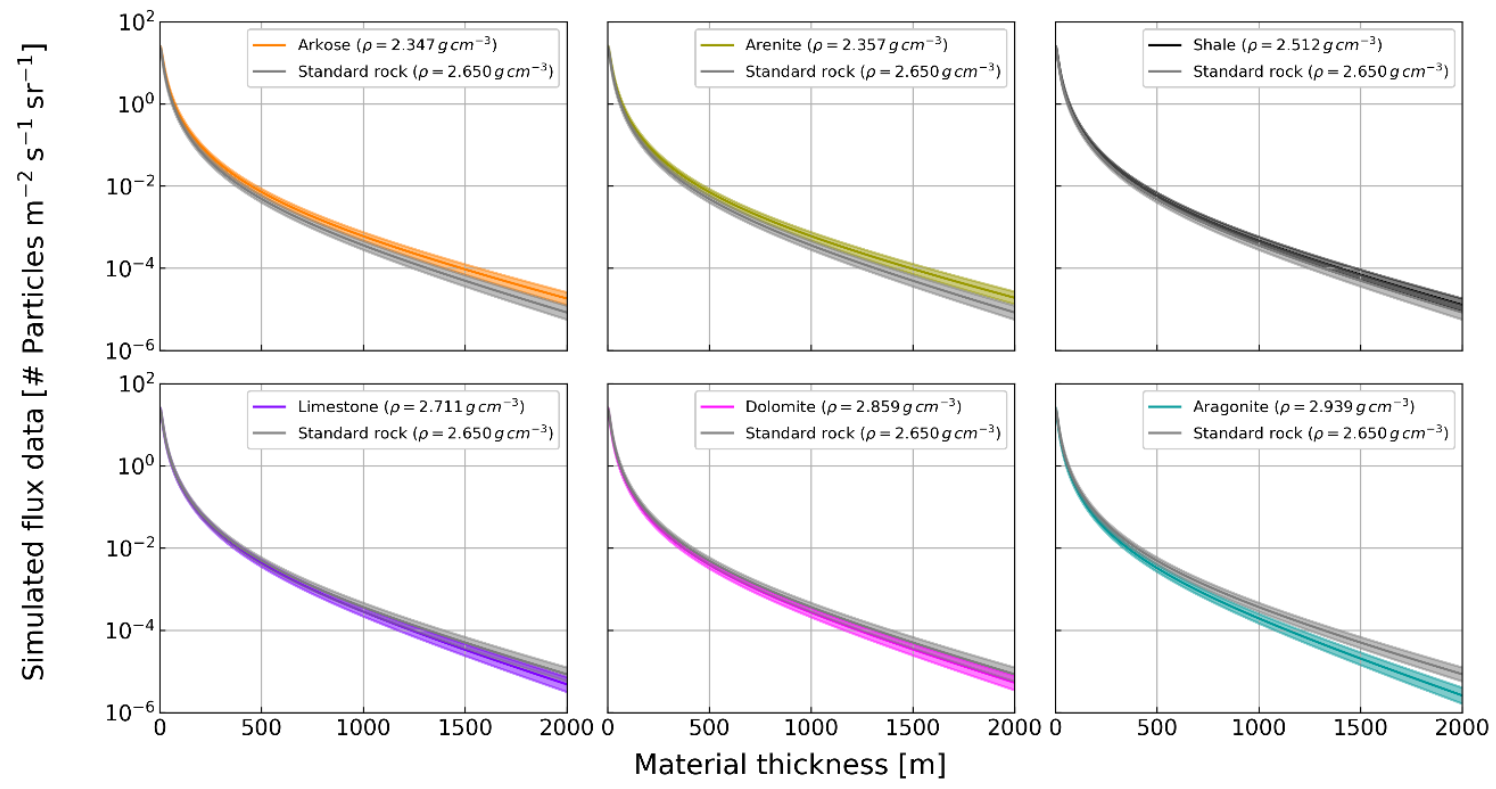

Figure S2-2: Simulated muon intensity vs. thickness of the six sedimentary rocks from Table 1 and standard rock. The mean flux is indicated by a bold line and $1 \sigma$ bounds are indicated by the shaded area. 


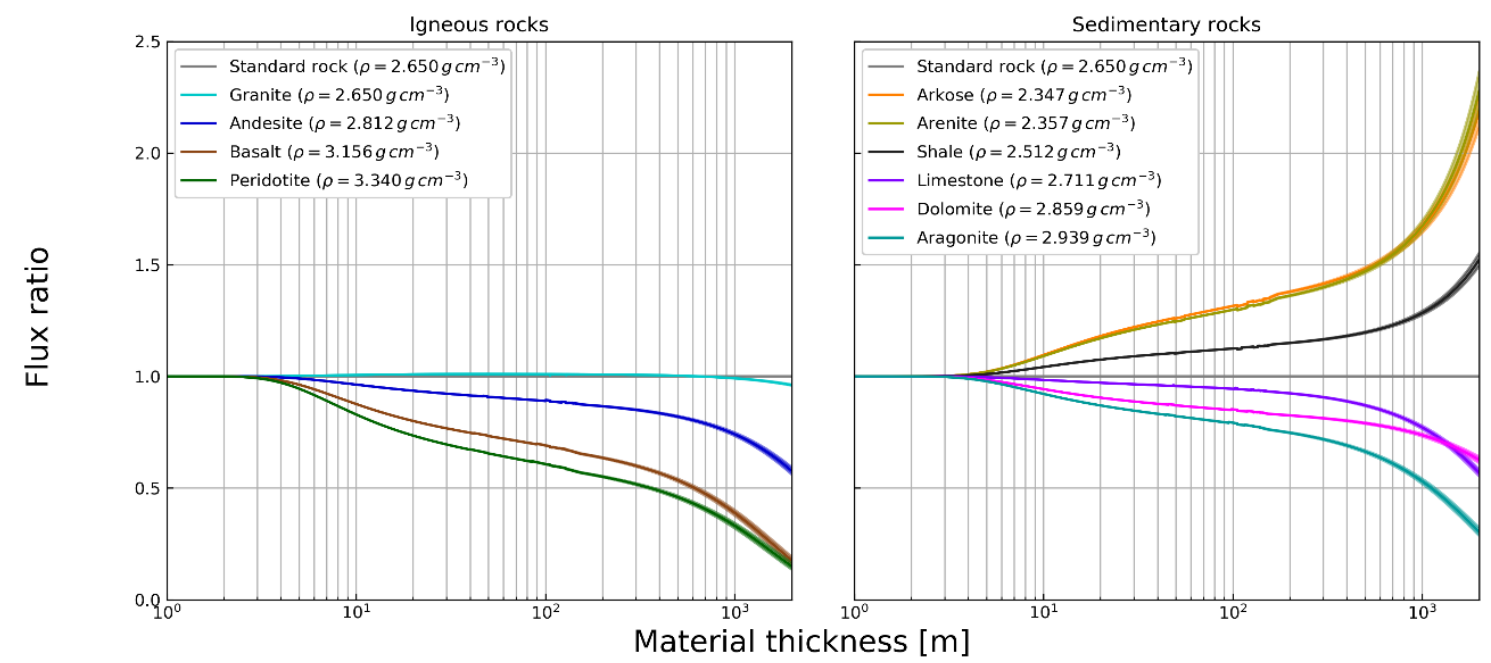

Figure S2-3: Ratio of the calculated rock fluxes to a standard rock $\left(\rho_{S R}=2.650 \mathrm{~g} \mathrm{~cm}^{-3}\right)$ muon flux for the rocks reported in Table 1 as a function of rock thickness.

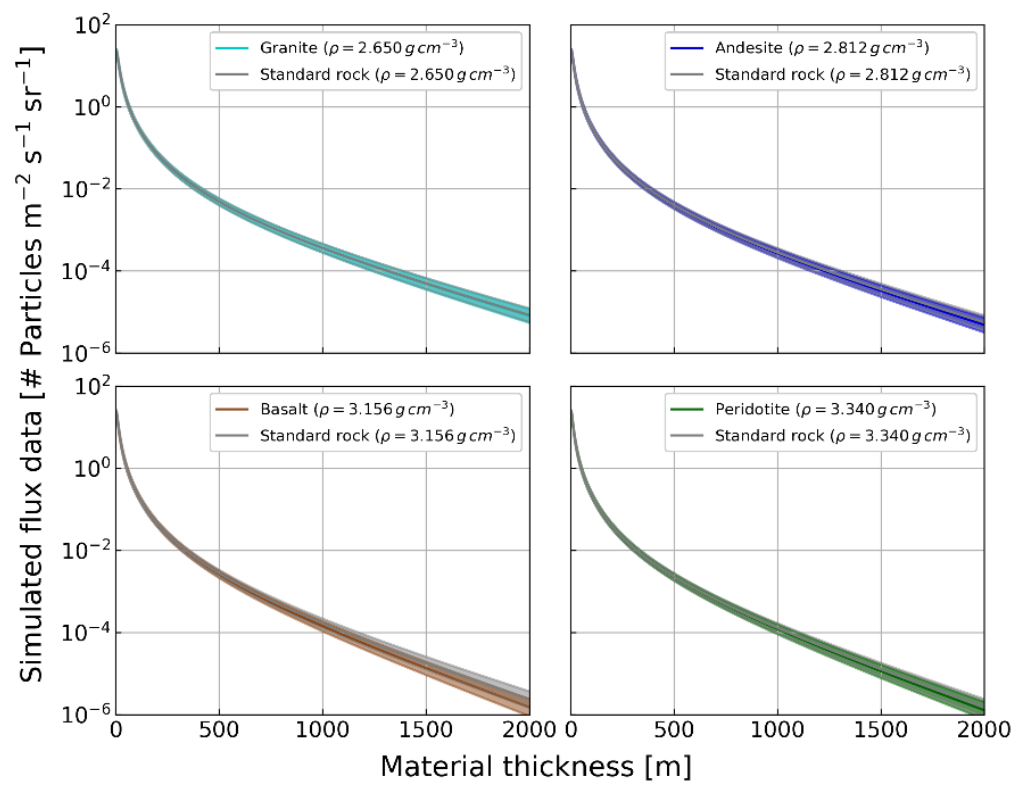

Figure S2-4: Simulated muon intensity vs. thickness of the four igneous rocks from Table 1 and a density modified standard rock. The mean flux is indicated by a bold line and $1 \sigma$ bounds are indicated by the shaded area.
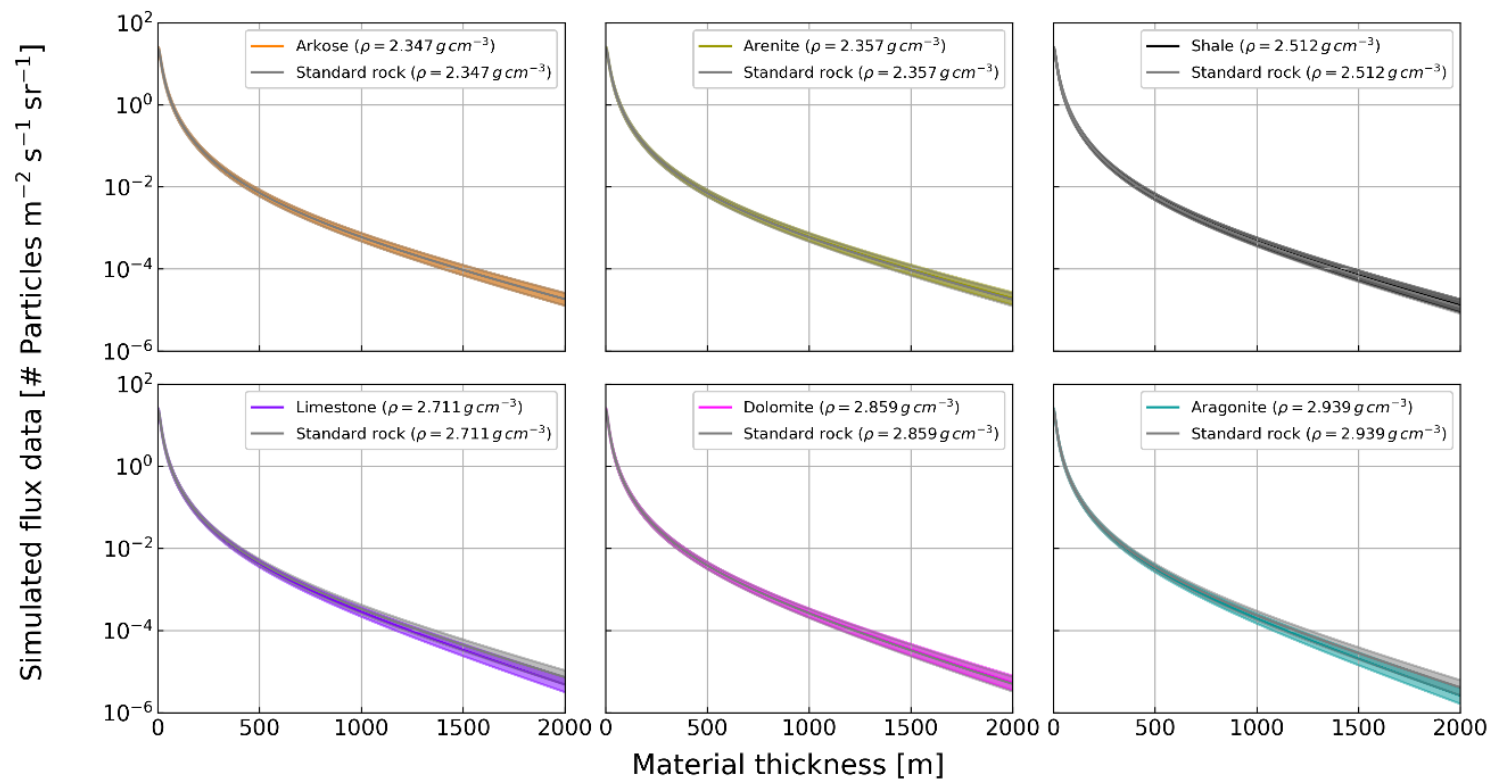

Figure S2-5: Simulated muon intensity vs. thickness of the six sedimentary rocks from Table 1 and a density modified standard rock. The mean flux is indicated by a bold line and $1 \sigma$ bounds are indicated by the shaded area. 


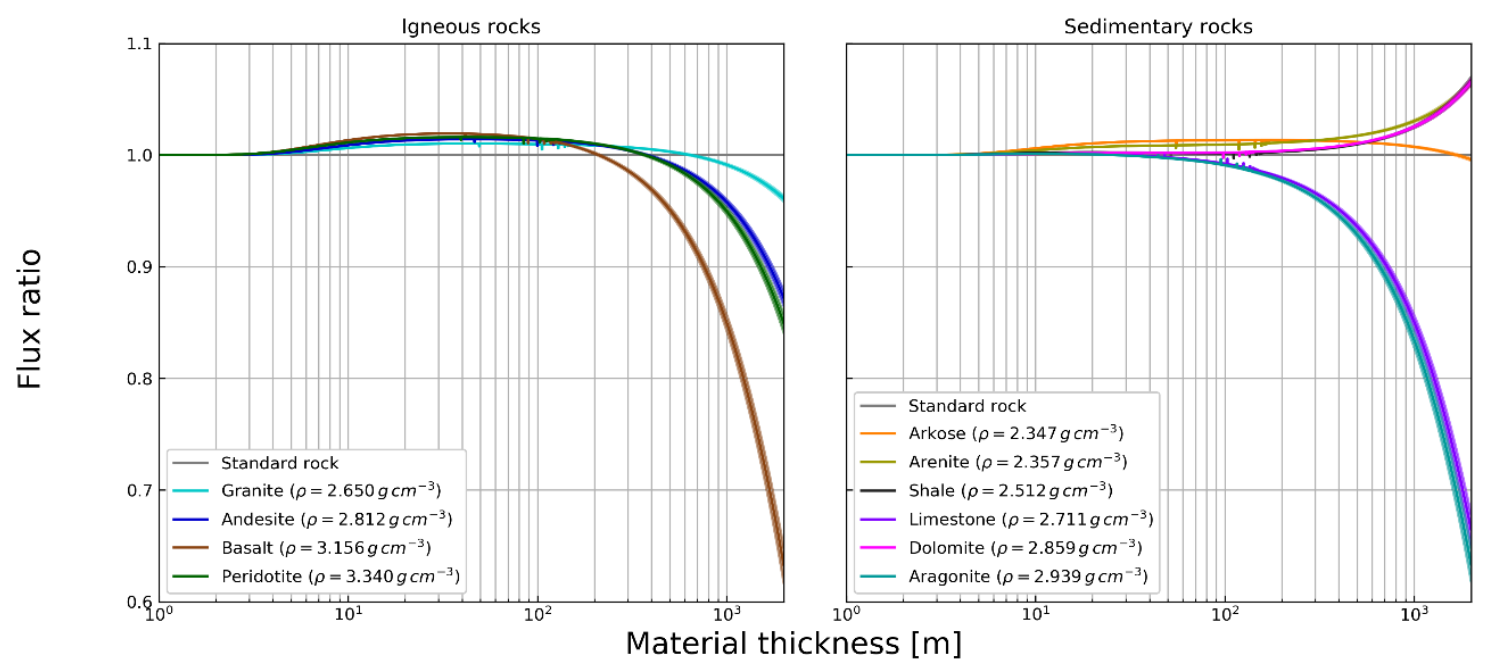

Figure S2-6: Ratio of the simulated rock fluxes to a standard rock muon flux with the same density as the rock $\left(\rho_{S R}=\rho_{R o c k}\right)$ for all the lithologies in Table 1 as a function of rock thickness.

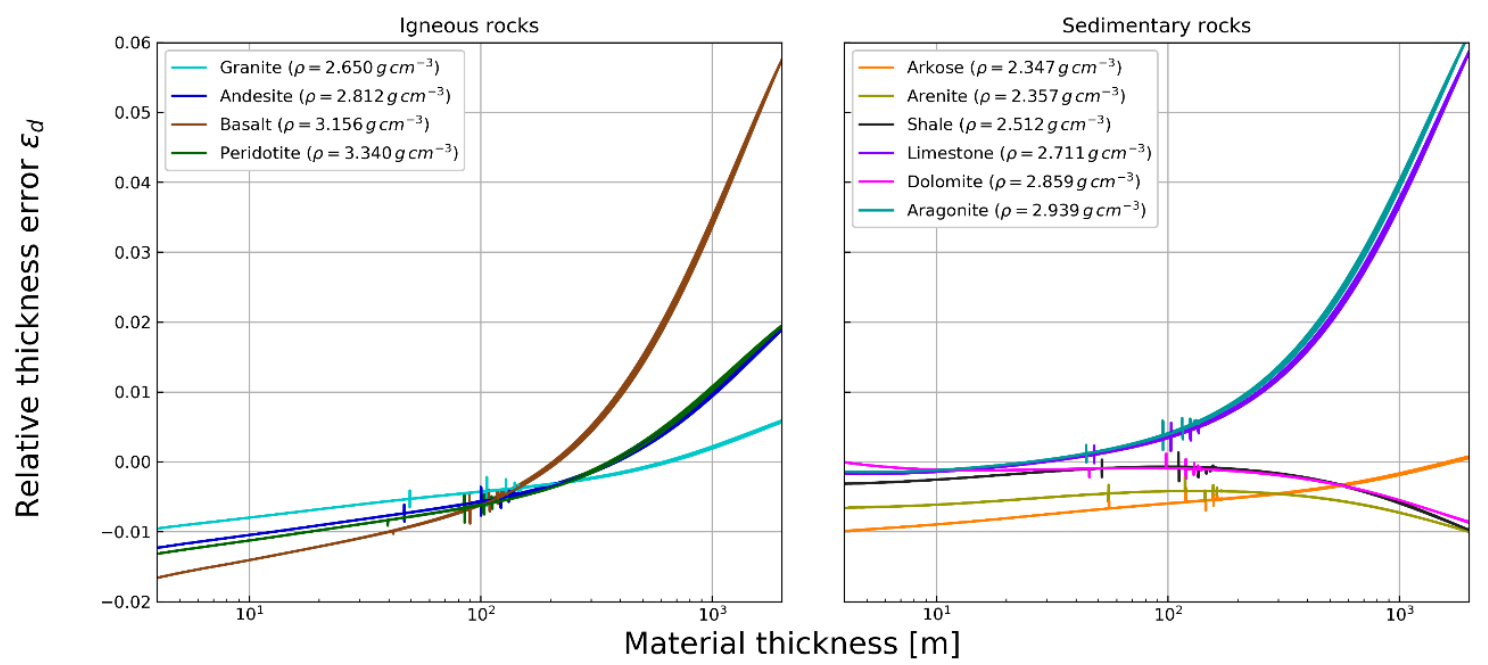

Figure S2-7: Relative error, which is made in the thickness estimation of a block of rock by assuming a density modified standard rock versus the actual rock thickness.

Most notably this flux model exhibits lower fluxes at low energies. However, this difference does not project into Fig. S2-3, S2-6 or S2-7, as the fluxes for both simulations have been changed equally. Thus, the rock composition effect is not affected by the lower flux from the flux model. The small numerical instabilities, well visible in Fig. S2-7, can be explained by the fact that the flux model by Tang et al. (2006) is a composite function for different energy ranges. At the transitions of one energy domain into another a jump might occur, which is visible as a peak in its ratio.

\section{References}

Reyna, D.: A Simple Parameterization of the Cosmic-Ray Muon Momentum Spectra at the Surface as a Function of Zenith Angle, arXiv Prepr. hep-ph/0604145 [online] Available from: http://arxiv.org/abs/hep-ph/0604145, 2006.

Tang, A., Horton-Smith, G., Kudryavtsev, V. A. and Tonazzo, A.: Muon simulations for Super-Kamiokande, KamLAND, and CHOOZ, Phys. Rev. D - Part. Fields, Gravit. Cosmol., 74, 1-34, doi:10.1103/PhysRevD.74.053007, 2006. 